\title{
Colangiopatía asociada a SIDA: Cuando la sola imagen no basta
}

\author{
Dr. Leonardo Lidid $A^{(1)}$, Int Camilo Apey $R^{(2)}$.
}

1. Profesor Asistente de Radiología. Campus Occidente, Facultad de Medicina, Universidad de Chile, Hospital San Juan de Dios. Radiólogo Servicio de Imagenología Hospital DIPRECA. Chile.

2. Interno de Medicina. Campus Occidente, Facultad de Medicina, Universidad de Chile, Hospital San Juan de Dios. Chile.

\section{AIDS cholangiopathy: When images in isolation are not enough}

\begin{abstract}
In HIV-infected patients, liver and biliary tract may be affected by different entities, such as AIDScholangiopathy, which is usually associated with CD4+T lymphocytes count below $100 \mathrm{cells} / \mathrm{mm}^{3}$ along with non-specific symptoms. Although imaging studies play a major diagnostic role they should always be evaluated according to patients clinical context. While ultrasound and CT scans provide relevant diagnostic information, specialized studies such as MRI and MRCP have become increasingly valuable due to their ability to demonstrate parietal as well as stenotic biliary changes. Despite ERCP remains the gold-standard for diagnosis of AIDS-related cholangiopathy, currently it is usually reserved to clearly exclude the presence of malignancy, or as a specific invasive therapeutic procedure, when indicated.

Key words: AIDS, Biliary tract diseases, Endoscopic retrograde cholangiopancreatography (ERCP), HIV, Magnetic resonance imaging (MRI), Sclerosing cholangitis.
\end{abstract}

Resumen: En pacientes infectados con VIH, el hígado y las vías biliares pueden presentar compromiso de distinta índole. Dentro de éstos, la colangiopatía asociada al SIDA se presenta con una sintomatología más bien inespecífica y habitualmente con recuentos de linfocitos $T C D 4<100 / \mathrm{mm}^{3}$. En este escenario, si bien los estudios de imagen juegan un rol muy relevante, éstos siempre deben ser evaluados en relación al contexto clínico del paciente. Si bien la ecografía y la TC aportan valiosa información en el diagnóstico, en la actualidad la resonancia magnética de abdomen combinada con colangioresonancia tienen gran utilidad, por ser capaces de demostrar tanto las alteraciones parietales, como los cambios morfológicos estenóticos biliares de esta patología. Si bien la ERCP aún conserva su valor de gold standard para el diagnóstico, actualmente la tendencia se dirige a reservarla para el descarte de neoplasias, en casos dudosos o como procedimiento terapéutico invasivo específico, cuando sea indicado.

Palabras clave: Colangiopancreatografía endoscópica retrógrada (ERCP), Colangitis esclerosante, Patología de la vía biliar, Resonancia magnética (RM), SIDA, VIH.

Lidid L, et al. Colangiopatía asociada a SIDA: Cuando la sola imagen no basta. Rev Chil Radiol 2012; 18(4): 184-189. Correspondencia: Dr. Leonardo Lidid A. / leolidid@gmail.com

Trabajo recibido el 20 de septiembre de 2012, aceptado para publicación el 25 de noviembre de 2012.

\section{Introducción}

EI VIH/SIDA es una epidemia de nivel mundial de la cual Chile no está ajeno. En nuestro país, en diciembre de 2008 existía un total de 20.099 casos notificados, estimándose que a nivel nacional unas 50.000 personas vivirían infectadas con este virus ${ }^{(1)}$. Aunque existen importantes avances y conocimiento en el desarrollo y tratamiento de esta patología, hoy en día, en Chile no es raro que estos pacientes sean pesquisados en estadios avanzados de su enfermedad. Es así como la tasa de notificación de VIH en Chile el 2002 fue de 5,6 pacientes por 100.000 habitantes, mientras que la de pacientes en etapa SIDA fue de aproxi- madamente 3 casos por 100.000 habitantes $^{(2)}$. Si observamos el contexto mundial, apreciaremos que la epidemiología de esta enfermedad ha ido cambiando, ya que gracias al desarrollo y difusión de las terapias antiretrovirales altamente efectivas, ha ocurrido un significativo aumento en la expectativa de sobrevida de estos pacientes ${ }^{(3)}$. De esta forma patologías asociadas que antes probablemente permanecían subdiagnosticadas, en la actualidad se hacen más evidentes.

Dentro del amplio rango de manifestaciones de esta enfermedad, el compromiso biliar y hepático no es infrecuente ${ }^{(4)}$, observándose especialmente, aunque no exclusivamente, en pacientes severamente 
inmunosuprimidos ${ }^{(5)}$. En este grupo de enfermedades destaca la colangiopatía asociada al SIDA, dado el poco conocimiento que existe de ella y la similitud de sus patrones imagenológicos con otro tipo de patologías. Por estas razones exponemos el siguiente caso y realizamos una revisión de la literatura con énfasis en las distintas formas de presentación y sus principales diagnósticos diferenciales.

\section{Caso clínico}

Paciente de sexo masculino, de 21 años de edad, VIH positivo, diagnosticado a la edad de 19 años, con falta de apego a terapia antirretroviral (TARV) y con múltiples hospitalizaciones por complicaciones de su cuadro de base, destacando entre ellos varios episodios de neumonía, candidiasis orofaríngea y un sarcoma de Kaposi diseminado.

En la presente hospitalización ingresa por cuadro de dolor abdominal en hipocondrio derecho asociado a ictericia y CEG. Dentro de los exámenes realizados destacan un perfil hepático alterado con patrón

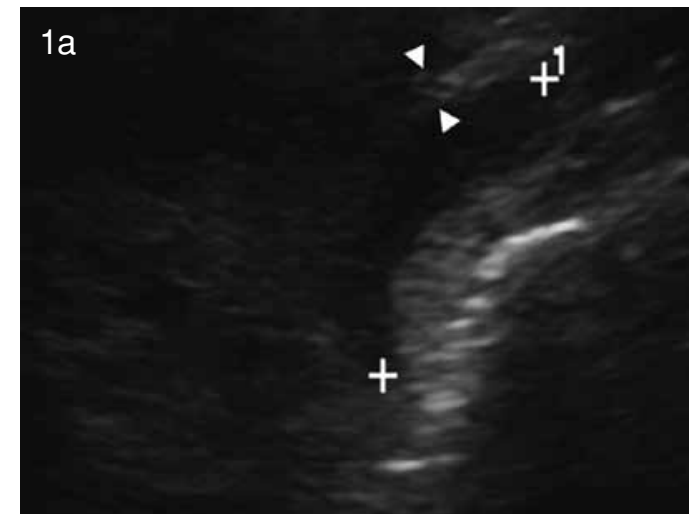

colestásico (Fosfatasas alcalinas y Gama glutamil transferasa [GGT] sobre 8 veces valor de referencia) y pancitopenia en el estudio hematológico. Tres meses previos a esta hospitalización el recuento de linfocitos T CD4 fue menor a $20 / \mathrm{mm}^{3}$.

En ecotomografía abdominal se describen hepatoesplenomegalia con varias lesiones focales hepáticas de pequeño tamaño relacionadas a su sarcoma diseminado, apreciándose también una vesícula biliar con paredes engrosadas en forma difusa, sin litiasis y una vía biliar extrahepática sin dilatación, pero con marcado engrosamiento parietal (Figura 1).

En los exámenes tomográficos se observan similares hallazgos, haciéndose más evidente el edema vesicular y el engrosamiento anular captante de la vía biliar, asociados a la presencia de otros fenómenos vinculados a su enfermedad sarcomatosa de base (Figura 2). Hasta ese momento, los hallazgos biliares presentes en el conjunto de los estudios imagenológicos, fueron interpretados como una colangiopatía asociada a SIDA.

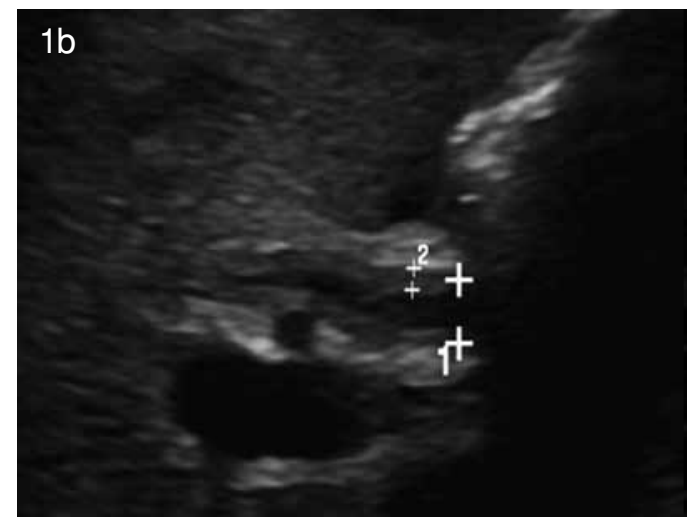

Figura 1. Afectación biliar y vesicular en la colangiopatía asociada a SIDA. Visión ecográfica. a) Imagen ecográfica de la vesícula biliar con engrosamiento parietal (cabezas de flecha). b) Corte ecográfico longitudinal de la vía biliar que muestra un calibre normal de ésta (señalada con el número 1) y un evidente engrosamiento parietal del hépato-colédoco (señalado con el número 2).
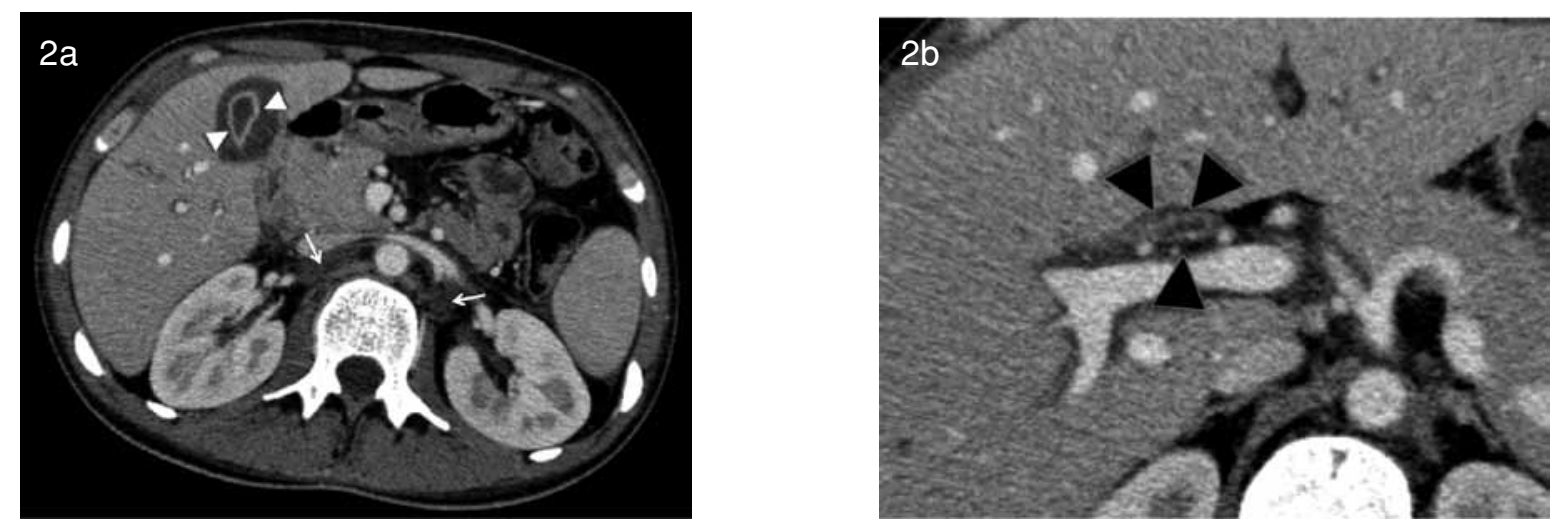

Figura 2. Cortes axiales de TC contrastado de abdomen en colangiopatía asociada a SIDA. A) En fase portal se evidencia edema parietal vesicular con impregnación mucosa de ésta (puntas de flecha). En el retroperitoneo empieza a insinuarse un conglomerado adenopático secundario a Sarcoma de Kaposi diseminado (flechas), el que se hace mucho más evidente a nivel pelviano (no mostrado). B) Engrosamiento parietal de la vía biliar, con significativa impregnación del medio de contraste (puntas de flecha). 
La resonancia magnética abdominal presenta hallazgos similares, llamando especialmente la atención en las secuencias colangiográficas, la existencia de un patrón arrosariado de la vía biliar intrahepática con ausencia de cálculos en su interior. Este estudio, realizado en otra institución, fue evaluado aisladamente sin contar con antecedentes ni exámenes previos, planteándose el diagnóstico de colangitis esclerosante (Figura 3). Por razones administrativas y sociales no se instauró terapia antirretroviral. En sucesivas hospitalizaciones posteriores persistían los hallazgos biliares descritos, con un severo progreso del compromiso visceral de su Sarcoma de Kaposi y de las infecciones oportunistas asociadas. El estado general del paciente se vio severamente comprometido, solicitando éste su alta voluntaria en la última hospitalización registrada.
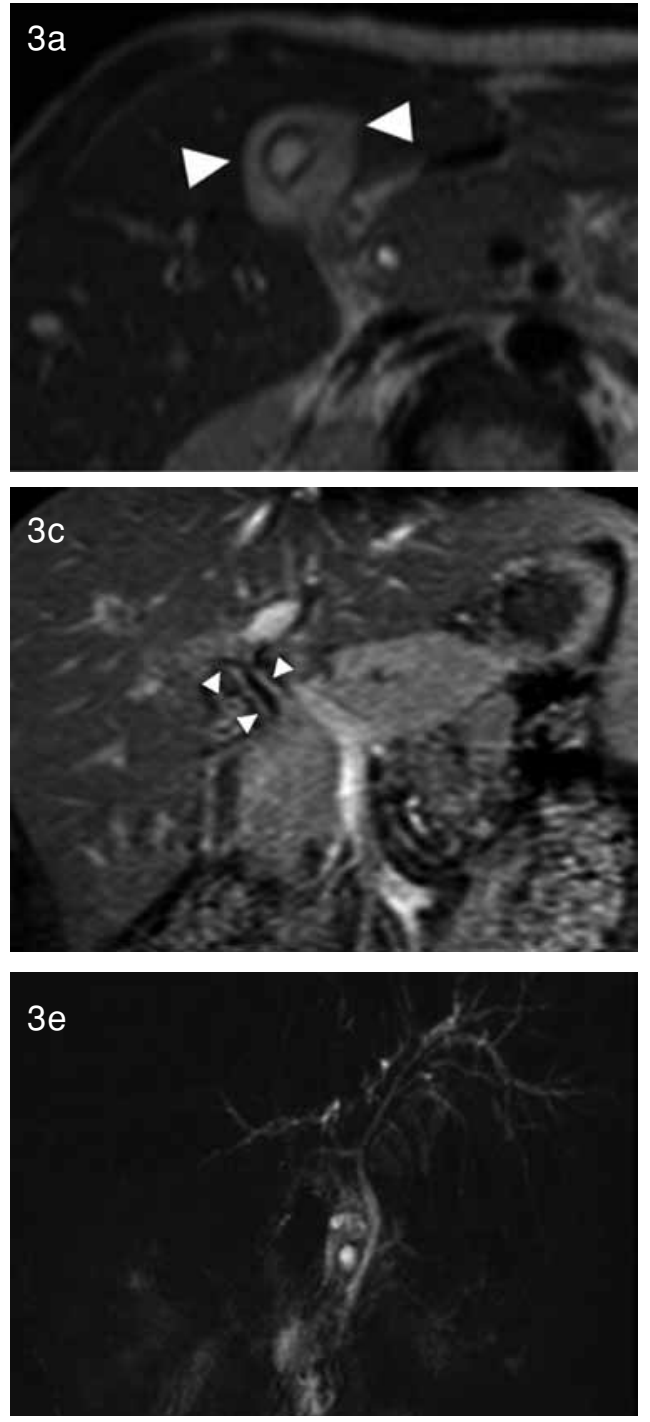

Discusión Los desórdenes biliares descritos en pacientes portadores del VIH pueden clasificarse dentro de tres grupos: patología biliar no asociada a $\mathrm{VIH}$ (por ejemplo, patología biliar litiásica), colecistitis alitiásica (CA) y colangiopatía asociada al SIDA (CAS) ${ }^{(4)}$. A continuación nos referiremos específicamente a la colangiopatía asociada al SIDA, entidad descrita por primera vez por Guarda, et al $1983^{(6)}$.

Epidemiología. Aunque la prevalencia exacta de la CAS es desconocida, un estudio realizado en India mostró una incidencia cercana al $1 \%$ en pacientes portadores del $\mathrm{VIH}^{(7)}$. Sin embargo, cuando se evalúan estas cifras hay que considerar el hecho que esta patología suele observarse con más frecuencia en pacientes que ya se encuentran en etapa $\operatorname{SIDA}^{(4)}$.
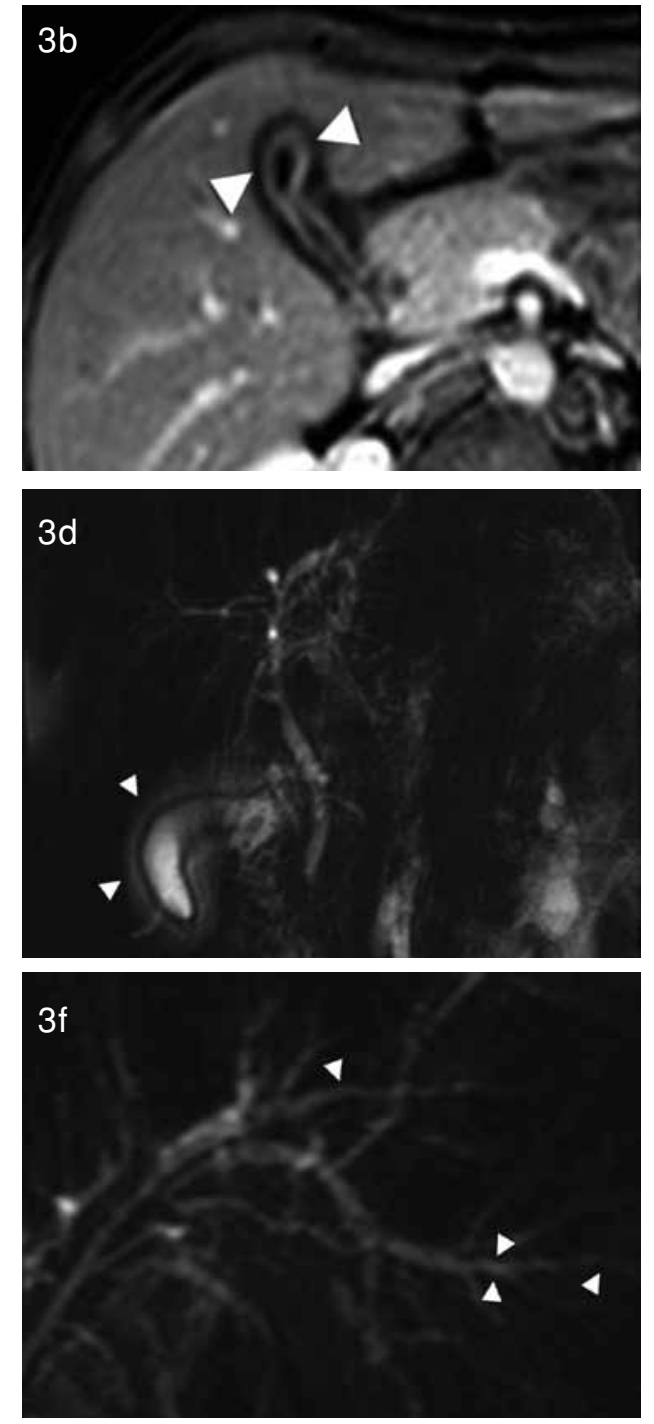

Figura 3. Resonancia magnética abdominal de colangiopatía asociada a SIDA. a) Secuencia T2 axial que muestra engrosamiento y edema parietal vesicular (cabezas de flecha). b) Adquisición axial T1 FAT-SAT en fase portal post inyección de gadolíneo en que se evidencian los mismos hallazgos y presencia de evidente impregnación mucosa del medio de contraste (cabezas de flecha). c) Adquisición coronal contrastada T1 FAT-SAT que muestra engrosamiento e impregnación parietal del colédoco. $d, e, f)$ Secuencias colangiográficas. En (d) se hace evidente el edema vesicular ya descrito (cabezas de flecha) y las alteraciones intrahepáticas de la vía biliar (arrosariamiento), los que son visibles de mejor manera en otra de las proyecciones ortogonales (e) y en una ampliación focalizada del árbol biliar izquierdo (cabezas de flecha) de esta misma imagen (f). 
Fisiopatología y patogenia. Se cree que el origen de la CAS se encuentra en infecciones oportunistas repetidas, pareciendo la vía biliar de pacientes VIH especialmente susceptible a éstas debido, entre otros factores, a una menor capacidad de reconocimiento de patógenos secundario a una disminución en la expresión de receptores Toll-like presentes en la vía biliar ${ }^{(8)}$.

Si bien existe una amplia gama de patógenos vinculados a esta patología, Cryptosporidium parvum y Citomegalovirus son los agentes más frecuentemente asociados al desarrollo de ésta(4).

Se ha propuesto que la patogenia esencial de este desorden consistiría en el desarrollo de estenosis de la vía biliar y de la papila de Vater tras recurrentes episodios inflamatorios de estas estructuras, aunque una acción directa del VIH también ha sido considerada $^{(9)}$. Las biopsias de la ampolla de Vater en general muestran infiltrados submucosos, inflamación periductal asociado a edema intersticial, infiltrados neutrofílicos e hiperplasia/dilatación de glándulas peri ductales ${ }^{(10)}$.

Clínica. La clínica de la colangiopatía por VIH es más bien inespecífica. La mayoría de los pacientes (90\%) se presenta con dolor en hipocondrio derecho $y / 0$ en epigastrio ${ }^{(4)}$. Otros síntomas incluyen náuseas, vómitos, fiebre y diarrea. La ictericia se ha descrito en $10 \%$ de los pacientes ${ }^{(11)}$, siendo un signo poco frecuente de encontrar.

Laboratorio. Suelen apreciarse alteraciones de las pruebas hepáticas, siendo lo más frecuente de encontrar un patrón colestásico, con elevación de la GGT y FA, encontrándose ésta última en torno a los 700-800UI/L. La mayoría de los pacientes presentan también un incremento discreto de las transaminasas y de la bilirrubina total, aunque pueden encontrarse pruebas de función hepática en rangos normales hasta en un $20 \%$ de los $\operatorname{casos}^{(4)}$.

Frecuentemente, aunque no exclusivamente, los pacientes con CAS se presenten con recuentos de linfocitos T CD4 $<100 / \mathrm{mm}^{3(11)}$. Aun así, la utilidad de utilizar el recuento de CD4 como factor pronóstico es discutido ${ }^{(4,12)}$.

Estudio por imágenes. El ultrasonido (US) representa el examen imagenológico más costo-efectivo para iniciar el estudio de pacientes con CAS con una sensibilidad entre $75-97 \%$ y especificidad de casi $100 \%$. Dentro de los hallazgos más frecuentes se incluyen dilatación del colédoco en el $70 \%$ de los casos y engrosamiento de la pared de éste en un $30 \%$ de los pacientes ${ }^{(4)}$. También puede apreciarse engrosamiento de la pared vesicu$\operatorname{lar}^{(9)}$ y una imagen nodular ecogénica en el extremo distal del colédoco, debido a edema de la Papila de Vater ${ }^{(13)}$. La tomografía computada (TC) de abdomen puede evidenciar dilatación de la vía biliar intrahepática de mejor manera que el ultrasonido, pero es menos sensible que este último en detectar engrosamiento y estenosis de la pared del colédoco ${ }^{(14)}$.

El examen más específico para el diagnóstico de CAS es la colangiopancreatografía endoscópica retrógrada (ERCP), la que además tiene la ventaja de resultar terapéutica en algunos pacientes. Si bien por ERCP existe una clasificación morfológica descriptiva que divide su presentación en cuatro categorías, para una mayor comprensión, los hallazgos por este método pueden ser agrupados en tres patrones morfológicos básicos que se pueden presentar en forma aislada 0 combinada entre sí (Figura 4) $)^{(3,9)}$ :

A) Patrón de colangitis esclerosante.

B) Estenosis papilar focal.

C) Estenosis segmentaria (larga) de la vía biliar extrahepática.
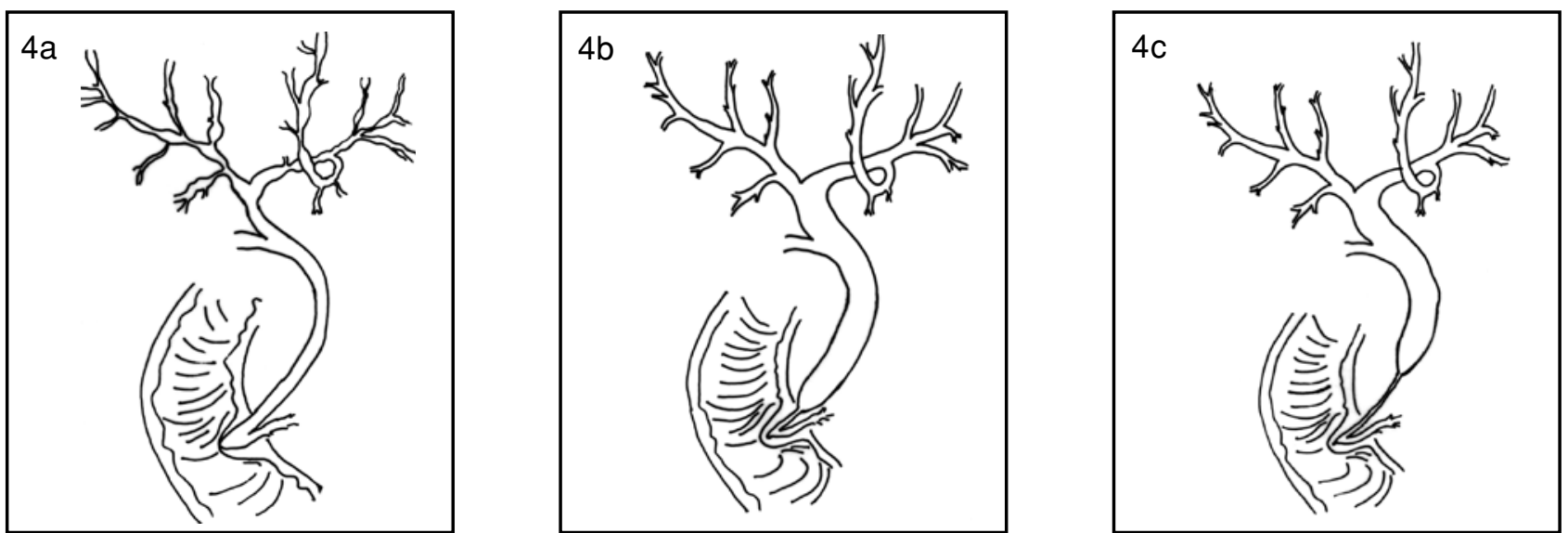

Figura 4. Esquema que representa los tres patrones morfológicos básicos de estenosis en la colangiopatía asociada al SIDA que se pueden presentar independientemente o combinados entre sí. a) Patrón intrahepático de colangitis esclerosante: múltiples focos de estenosis intrahepática con dilataciones segmentarias moderadas, dando un aspecto arrosariado a la vía biliar. Este patrón también puede incluir focos de estenosis de la vía biliar extrahepática. b) Estenosis papilar: dilatación de la vía biliar secundaria a estenosis en general poco pronunciada del colédoco papilar: Puede asociarse a moderada dilatación del conducto de Wirsung. c) Estenosis segmentaria larga (de 1-3 cm) de cualquier segmento de la vía biliar extrahepática: puede ser única o múltiple. 
La estenosis papilar es el evento aislado encontrado más frecuentemente (36\%). La combinación más común corresponde a la asociación de estenosis papilar y el patrón de colangitis esclerosante que se observa hasta en un $44 \%$ de los pacientes. El patrón más infrecuente es el de estenosis biliar larga extrahepática que alcanza un $5 \%$ de los $\operatorname{casos}^{(4,12)}$.

En la actualidad, el uso combinado de la resonancia magnética y la colangiopancreatografía por resonancia magnética (CPRM) son de gran utilidad para evaluar tanto patología biliar como patología del parénquima hepático ${ }^{(5)}$. En éstos se observan los mismos hallazgos de engrosamiento, edema y captación parietal biliar descritos en TC, pudiéndose agregar en las secuencias colangiográficas patrones morfológicos similares a los descritos en ERCP(15). El carácter no invasivo y el buen rendimiento general que exhibe la CPRM en la evaluación de alteraciones biliares, incluida la colangitis esclerosante, hace que algunos autores planteen su utilización con fines diagnósticos, reservando la ERCP para el tratamiento sintomático de la CAS o para el descarte de neoplasias mediante el estudio histológico o citológico directo ${ }^{(3,4)}$. Finalmente, existe consenso en que el diagnóstico definitivo de la CAS se logra mediante la adecuada interpretación de los antecedentes clínicos, hallazgos de laboratorio y patrones imagenológicos ${ }^{(4)}$.

Diagnósticos diferenciales. El principal diagnóstico diferencial de la CAS lo representa la colangitis esclerosante ${ }^{(15)}$, ya que el compromiso morfológico intrahepático puede ser indistinguible de la colangitis esclerosante primaria, tal como se evidencia en el caso descrito. Ambas patologías se caracterizan por estenosis focales de la vía biliar intercalados con dilataciones segmentarias moderadas, dando un aspecto arrosariado a la vía biliar intrahepática ${ }^{(13)}$. Para diferenciarlos, cabe tener en cuenta que los pseudodivertículos en las paredes del colédoco y la estenosis de alto grado de la vía biliar extrahepática son hallazgos típicos de la colangitis esclerosante ${ }^{(15)}$ mientras una dilatación ductal moderada asociada a márgenes irregulares y nódulos orienta más hacia $\mathrm{CAS}^{(13)}$. La colangitis piógena, otro diagnóstico diferencial, suele tener un contexto séptico diferente ${ }^{(16)}$ y se asocia a alteraciones parenquimatosas hepáticas (por ejemplo microabcesos peri-biliares o trastornos de la perfusión multifocales) de los que la colangiopatía asociada al SIDA carece.

La estenosis fibrosa del colédoco distal secundaria al paso de cálculos biliares o de pancreatitis crónica representa otra causa posible de algunos de los hallazgos imagenológicos descritos en $\mathrm{CAS}^{(15)}$. Para diferenciarlos, es importante revisar los antecedentes clínicos y estudios previos del paciente.

Por último, otros eventuales diagnósticos a tener en cuenta son la colecistitis alitiásica y el colangiocarcinoma. Sin embargo, el contexto clínico en éstos es bastante diferente. La primera, si bien también tiene una vesícula de paredes engrosadas, ocurre en pacientes sépticos graves, con falla multisistémica o signos de peritonitis. En el segundo caso, se aprecia obstrucción biliar progresiva en el tiempo y en la TC con contraste aparecen infiltrados ductales en forma de masa que invaden el parénquima hepático, con captación tardía del medio de contraste ${ }^{(4,15)}$.

Tratamiento y pronóstico. La terapia que puede ofrecerse a los pacientes portadores de CAS puede dividirse en tratamiento sintomático y en tratamiento modificador del curso de la enfermedad. Dentro del primer grupo podemos mencionar la esfinterotomía por medio de ERCP, la que provee alivio sintomático a cerca del $90 \%$ de los pacientes ${ }^{(4,11)}$ y se reserva para los casos con estenosis papilar demostrada ${ }^{(3)}$. La colocación de stents en la vía biliar o la dilatación de focos estenóticos mediante un balón podrían resultar exitosas en algunos pacientes, aunque no se cuenta con estudios randomizados que avalen su uso. Además, el ácido ursodeoxicólico ha sido usado exitosamente como tratamiento médico sintomático

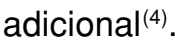

En cuanto al tratamiento modificador del curso de la enfermedad se ha descrito que el resultado de la CAS depende en gran medida del estatus del VIH subyacente ${ }^{(12)}$. La sobrevida media de los pacientes con CAS se ha descrito entre $9^{(4)}$ hasta 34 meses $^{(6)}$, lo que dependería en gran medida de la instauración de TARV luego del diagnóstico de CAS, si es que éste no se ha comenzado. El desarrollo de CAS durante el curso de TARV puede ser un signo de avance de la enfermedad, ya sea por inmunosupresión o por resistencia a la terapia anti retroviral. Por último, hay relativo consenso respecto que el tratamiento de las infecciones oportunistas subyacentes a la CAS no implica mejoría sintomática ni cambios evidentes en la morfología de la vía biliar(4).

Dado todo lo expuesto, es importante recalcar que la presencia de focos estenóticos biliares intra y/o extra hepáticos en un paciente VIH positivo hace necesario considerar la posibilidad de una colangiopatía asociada a SIDA, la que por sí misma puede constituir un indicador de progresión o gravedad de su enfermedad de base.

\section{Bibliografía}

1. Ministerio de Salud. Guía clínica Síndrome de Inmunodeficiencia Adquirida VIH/SIDA. 2nd Ed. Santiago: Minsal 2010: 9-10.

2. Ministerio de Salud, Departamento de Epidemiología. Situación epidemiológica de VIH/SIDA, Chile 19842004. Santiago: Minsal 2006: 2-3.

3. Tonolini M, Bianco R. HIV related/AIDS cholangiopathy: pictorial review with emphasis on MRCP findings and differential diagnosis. Clin Imag (2012); http://dx.doi. org/10.1016/j.clinimag.2012.03.008 (Article in press). 
4. Brun A, Pitchumoni C.S. Biliary Diseases in HIV. Practical gastroenterology 2010; 34(9): 24-32.

5. Bilgin M, Blaci C. N, Edrogan A, Javad M. A, Alkaade S, Rau W. Hepatobiliary and pancreatic MRI and MRCP findings in patients with HIV infections. AJR 2008; 191: 228-232.

6. Pitlik SD, Fainstein V, Ríos A, Guarda L, Mansell PW, Hersh EM. Cryptosporidial cholecystitis. N Engl J Med. Apr 21 1983: 308(16): 967.

7. Devarbhavi H, Dierkhising R, Kremers WK, Sandeep MS, Karanth D, Adarsh CK. Single-Center experience with drug-induced liver injury from India: causes, outcome, prognosis and predictors of mortality. Am J Gastroenterol 2010.

8. O'Hara SP, Small AJ, Gajdos GB, Badley AD, Chen XM, Larusso NF. HIV-1 Tat protein suppresses cholangiocyte toll-like receptor 4 expression and defense against Cryptosporidium parvum. J Infect Dis. Apr 15 2009; 199(8): 1195-1204.

9. Miller HF, Gore MR, Nemeck A.A, Fitzgerald W.S. Pancratobiliary manifestations of AIDS. AJR 1996; 166: 1269-1274.

10. Chen XM, La Russo NF. Cryptosporidiosis and pathogenesis of AIDS cholangiopathy. Semin Liver Dis 2002;
22(3): 277-289.

11. Bouche $H$, Housset $C$, Dumont JL, Carnot F, Menu $Y$, Aveline B, et al. AIDS-related cholangitis; diagnostic features and course in 15 patients. J Hepatol 1993; 17(1): 34-39.

12. Ko WF, Cello JP, Rogers SJ, Lecours A. Prognostic factors for the survival of patients with AIDS cholangiopathy. Am J Gastroenterol. 2003; 98(10): 2176-2181.

13. Da Silva F, Boudghene F, Lecomte I, Delage Y, Grange JD, Bigot JM. Sonography in AIDS-related cholangitis: prevalence and cause of an echogenic nodule in the distal end of the common bile duct. AJR 1993; 160: 1205-1207.

14. Nash JA, Cohen SA. Gallbladder and biliary tract disease in AIDS. Gastroenterol Clin North Am. 1997; 26(2): 323-335.

15. Brooke Jeffery R. Colangiopatía del SIDA. Federle M, Brooke Jeffery R, Woodward P, Borhani A. Abdomen: Diagnóstico por imagen. Segunda edición. Madrid: Marbán Libros; 2011. Capítulo III - 2. 26-29.

16. Van Erpecum K. Complications of bile-duct stones: acute cholangitis and pancreatitis. Clin Gastroenterol 2006; 20(6): 1139-1152. 